\section{Review: walking aids, chest-wall vibration, and neuroelectrical muscle stimulation relieve breathlessness in COPD}

\section{QUESTION}

In advanced disease, do non-pharmacological and noninvasive interventions relieve breathlessness?

\section{REVIEW SCOPE}

Studies selected evaluated non-pharmacological or noninvasive interventions for breathlessness in patients with dyspnoea because of advanced lung cancer, chronic obstructive pulmonary disease (COPD), interstitial lung disease, chronic heart failure, or motor neurone disease. Outcomes included subjective measures of breathlessness.

\section{REVIEW METHODS}

Medline; EMBASE/Excerpta Medica; CINAHL; Cochrane Central Register of Controlled Trials; Cochrane Pain, Palliative, and Supportive Care Trials Register; Cochrane Database of Systematic Reviews; and 5 other databases (all to Jun 2007); websites; and reference lists were searched. Authors were contacted. 45 randomised controlled trials (RCTs, $\mathrm{n}=2282$, mean age $69 \mathrm{y}$ based on 37 RCTs) and 2 controlled clinical trials $(\mathrm{n}=250)$ met the selection criteria. Meta-analysis was not done because of heterogeneity between studies. Most studies had low quality based on the Oxford Quality Scale but high quality based on the Edwards scale.

\section{MAIN RESULTS}

The table summarises the findings of RCTs of patients with COPD (35 of 47 studies).

\section{CONCLUSION}

Walking aids, chest-wall vibration, and neuroelectrical muscle stimulation relieve breathlessness in chronic obstructive pulmonary disease.

\section{ABSTRACTED FROM}

Bausewein C, Booth S, Gysels M, et al. Non-pharmacological interventions for breathlessness in advanced stages of malignant and non-malignant diseases. Cochrane Database Syst Rev 2008;(2):CD005623.

Correspondence to: Dr C Bausewein, King's College London, London, UK claudia.bausewein@kcl.ac.uk

Source of funding: Cicely Saunders International.

- Clinical impact ratings: Cardiology 4/7; Oncology 7/7; Palliative care 6/7; Respirology 6/7

\begin{tabular}{lll}
\multicolumn{2}{l}{ Various interventions $v$ no intervention or } & placebo for breathlessness in chronic obstructive pulmonary disease \\
\hline Intervention & Number of trials $(\mathbf{n})$ & Effects on breathlessness at 1 day to 1 year* \\
\hline Acupuncture & $2(62)$ & Improved (1 trial, low-quality evidence) \\
Acupressure & $2(75)$ & Improved (low-quality evidence) \\
Distractive auditory stimuli (music) & $6(135)$ & No difference (4 trials) \\
Relaxation & $3(72)$ & No difference (2 trials) \\
Walking aids & $7(201)$ & Improved (5 trials) \\
Chest-wall vibration & $4(51)$ & Improved (3 trials) \\
Neuroelectrical muscle stimulation & $3(50)$ & Improved \\
Fan & $1(17)$ & No difference \\
Counselling & $1(177)$ & No difference \\
Breathing training & $2(99)$ & Improved (1 trial) \\
Case management & $1(66)$ & Improved \\
Psychotherapy & $2(83)$ & Improved (1 trial)
\end{tabular}

B reathlessness is a common and distressing symptom for people with advanced illness. The review by Bausewein et al of nonpharmacological interventions is an important adjunct to reviews of pharmacological management of breathlessness. ${ }^{1}$ Although a rigorous assessment was done, the limited number of studies, variable quality, and heterogeneity of interventions and outcomes made this a challenging synthesis. The multidimensional aspects of breathlessness in advanced disease are represented in the breadth of non-pharmacological interventions included in the review. Studies that were of sufficient quality to provide recommendations for practice included only patients with COPD and cannot be assumed to apply to the wider review population of patients with malignant and non-malignant conditions.
The effect of individual study quality on practice implications has to be tempered against the rea world constraints of different practice settings, resource limitations, variable costs, and patient preferences. For example, studies of chest-wall vibration were done in the laboratory, making translation to practice unclear. Breathing retraining was graded as medium quality, but the different breathing strategies used in the 3 studies limit any specific direction for practice. In contrast, walking aids, which were shown to clearly benefit patients with COPD, can be used in many practice environments. Neuroelectrical muscle stimulation of leg muscles is another well-tolerated intervention with potential for wider use. Despite having only lowquality evidence, some interventions are worth considering for use in practice. The crossover RCT of a hand-held fan showed significant improvement in breathlessness in a mixed population of patients with cardiopulmonary disease and is a practical, econom ical approach.

The review by Bausewein et al highlights the need for high-quality research that reflects the realities of people with advanced diseases that cause breathlessness and those who care for them.

Della Roberts, CNS End of Life Care

Fraser Health

Surrey, British Columbia, Canada

1. Jennings $\mathbf{A L}$, Davies $A N$, Higgins JP, et al. Thorax 2002;57:939-44.

2. Galbraith S, Perkins P, Lynch A, et al. Does a handheld fan improve intractable breathlessness? Proceeding EAPC Research Forum. 29-31 May 2008 Trondheim, Norway. 